\title{
Roles of Matrix Metalloproteinase-9 in Cancer Metastasis
}

\author{
Hyereen Kang ${ }^{1}$ and Sung-Wuk Jang ${ }^{1,2, \dagger}$ \\ ${ }^{I}$ Department of Biomedical Sciences, University of Ulsan College of Medicine, Seoul 138-736, Korea \\ ${ }^{2}$ Department of Biochemistry and Molecular Biology, University of Ulsan College of Medicine, \\ Seoul 138-736, Korea
}

\begin{abstract}
Matrix metalloproteinases (MMPs), also called matrixins, function in the extracellular environment of cells and degrade both matrix and non-matrix proteins. They are multidomain proteins and their activities are regulated by tissue inhibitor of metalloproteinases (TIMPs). The uncontrolled regulation of MMPs is involved in various pathologic processes, such as tumor invasion, migration, host immune escape, extravasation, angiogenesis, and tumor growth. Especially, matrix metalloproteinase-9 (MMP-9) is one of the metastasis-accelerating genes involved in metastasis of various types of human cancers. Here, we review the member of MMP family and discusses their domain structure and function, enzyme activation, the mechanism of inhibition by TIMPs. In particular, we focus the role of MMP-9 in relation to cancer metastasis.
\end{abstract}

Key Words: Extracellular proteinases, Matrix metallopproteinase-9, Metastasis, Invasion

\section{INTRODUCTION}

Metastasis is a complex series of steps in which cancer cells leave the original tumor site and migrate to other tissues of the body through the bloodstream or the lymphatic system (Nguyen and Massague, 2007). Cancer occurs after a single cell in a tissue is progressively genetically damaged to produce cells with uncontrolled proliferation. In general, malignant cells break away from the primary tumor and attach to and degrade proteins that form the surrounding extracellular matrix (ECM), which separates the tumor from neighboring tissues (Nguyen and Massague, 2007; Pantel and Brakenhoff, 2004). By degrading these proteins, cancer cells are able to breach the ECM and escape. Proteases of

*Received: May 29, 2014 / Revised: August 19, 2014

Accepted: August 20, 2014

${ }^{\dagger}$ Corresponding author: Sung-Wuk Jang. Department of Biochemistry and Molecular Biology, University of Ulsan College of Medicine, Seoul 138-736, Korea.

Tel: +82-2-3010-2088, Fax: +82-2-3010-2098

e-mail: swjang@amc.seoul.kr

C The Korean Society for Biomedical Laboratory Sciences. All rights reserved. the matrix metalloproteinase (MMP) family are involved in the breakdown of ECM in normal physiological processes, such as embryonic development, reproduction, angiogenesis, bone development, wound healing and cell migration, as well as in pathological processes, such as arthritis and metastasis (Van den Steen et al., 2002; Vandooren et al., 2013). Most MMPs are secreted as an inactive form which are activated when cleaved by extracellular proteinases.

MMP-9 plays a central role in tumor progression, including stromal remodeling, eventually metastasis, rheumatoid arthritis, arteriosclerosis and angiogenesis (Farina and Mackay, 2014; Kupferman et al., 2000). MMPs such as MMP-9 are involved in the development of several human malignancies, as degradation of collagen IV in basement membrane and extracellular matrix facilitates tumor progression, including invasion, metastasis, growth and angiogenesis (Groblewska et al., 2012). The purpose of this review is to examine the role of MMP-9 in relation to cancer metastasis. The regulatory mechanisms controlling these enzymes will also be discussed, particularly the tissue inhibitors of metalloproteinase (TIMPs). 


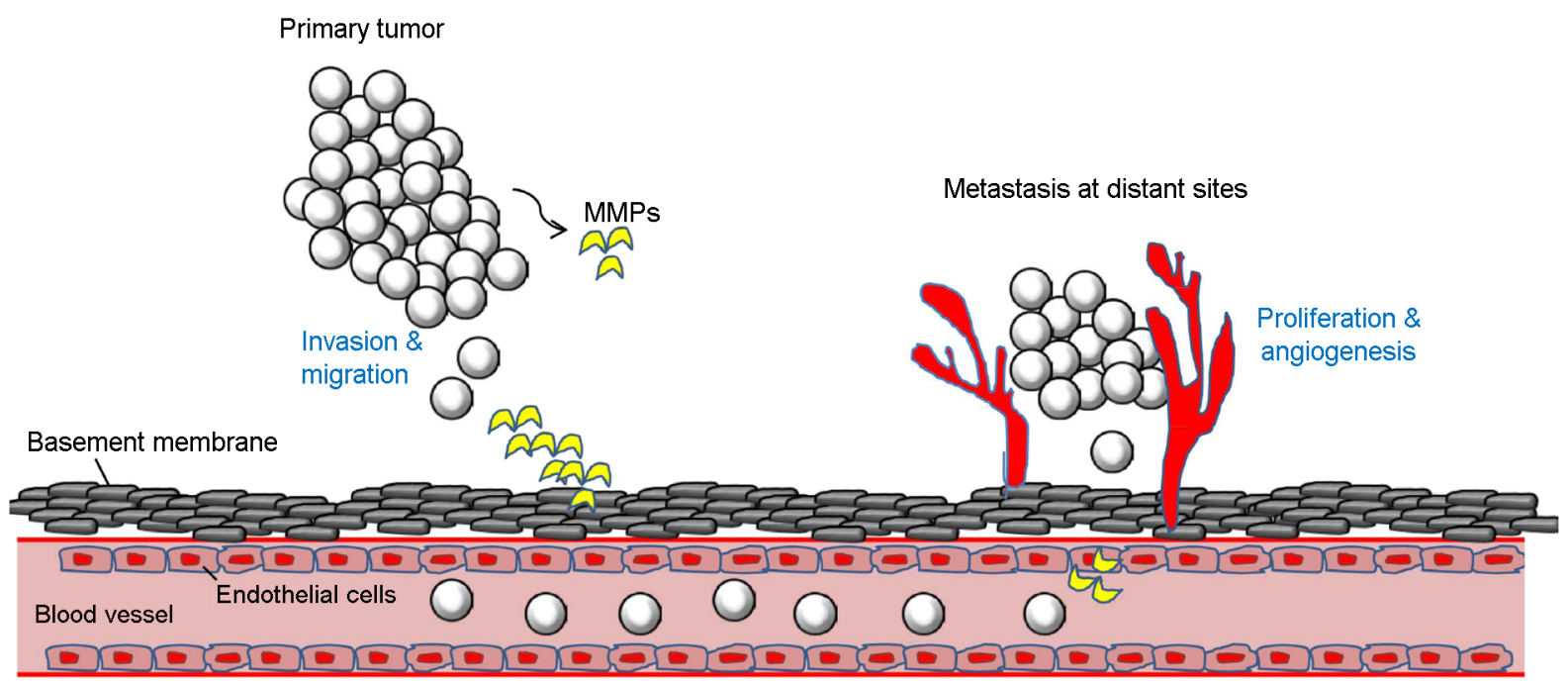

Circulation

Fig. 1. Metastasis of tumors. The sequential steps of metastatic cascade. Cancer surrounded by an intact basement membrane. Invasion requires reversible changes in cell-cell and cell-extracellular matrix adherence, destruction of protein in the matrix and stroma. Metastasizing cells can enter the circulation and extravasation of the circulatory system. Metastatic colonization of the distant site progresses through tumor cells.

\section{Tumor cell metastasis}

Metastasis is a complex multi-step process and, in the cascade of metastasis and invasion of the basement membrane and extracellular matrix by tumor cells is thought to be one of the most critical steps (Pantel and Brakenhoff, 2004; Roeb and Matern, 2001). This process is mediated by extracellular proteinases. Extracellular proteinases are essential for several developmental and disease-related conditions. MMPs consist of a family of zinc-dependent ECM remodeling endopeptidase implicated in physiological processes including wound healing, organogenesis, and pathological conditions, such as inflammation, vascular disorders, and carcinogenesis (Egeblad and Werb, 2002; Lynch and Matrisian, 2002; Sternlicht and Werb, 2001). During metastasis, there are a variety of interactions among cancer cells, the ECM and barriers (Leber and Efferth, 2009).

Cancer cells cross the epithelial basement membrane and invade the surrounding stroma, and then they enter blood vessels of lymphatics, extravasate and establish new proliferating colonies (Hanahan and Weinberg, 2000; Kim et al., 2004; Pantel and Brakenhoff, 2004). Metastasis consists of sequential steps: escape of cells from the primary tumor, intravasation (entry of cells into the blood circulation), survival and transport in the circulation, arrest in distant organs, extravasation (escape of cells from the circulation), and growth of cells to form secondary tumors in the new organ environment (Fig. 1) (Leber and Efferth, 2009; Murphy, 2008; Pantel and Brakenhoff, 2004). Angiogenesis, the development of new blood vessels, is required for the primary and metastatic tumors to grow beyond a minimal size and evasion of immune eradication is necessary at several steps (Fig. 1) (Leber and Efferth, 2009; Pantel and Brakenhoff, 2004).

Critical molecules involved in this progression are MMPs because they degrade various cell adhesion molecules, thereby modulating cell-cell and cell-ECM interactions (Lynch and Matrisian, 2002; Vandooren et al., 2013). The ECM is a dynamic structure that orchestrates the conduct of the cancer cells by interacting with them (Gialeli et al., 2011). The proteolytic activity of MMPs is required for a cancer cell to degrade physical barriers during local expansion and intravasation at nearby blood vessels, extravasation, and invasion at a distant location (Murphy, 2008). The 
Table 1. The matrix metalloproteinase family. Matrix Metalloproteinases (MMPs) are a large group of zinc-dependent endogenous proteolytic enzymes that are able to degrade all components of the extracellular matrix (ECM). The MMP family share several similarities in terms of their structure, regulation and function. Their structure commonly includes three peptide domains: a pro-peptide domain, a zinc-containing catalytic domain and (with the exceptions of MMP-7 and MMP-26) a hemopexin-like C-terminal domain, which determines their substrate specificity. Although MMPs have overlapping substrate specificities, they are divided into five groups with respect to their preferential degradation of different matrix substrates (matrilysin, collagenases, gelatinases, stromelysin and membrane MMPs (MT-MMPs).

\begin{tabular}{|c|c|c|}
\hline $\begin{array}{c}\text { MMP } \\
\text { designation }\end{array}$ & Structural class & Common names \\
\hline MMP-1 & Simple hemopexin domain & Collagenase-1, interstitial collagenase, fibroblasta collagenase, tissue collagenase \\
\hline MMP-2 & Gelatin-binding & Gelatinase $\mathrm{A}, 72-\mathrm{kDa}$ gelatinase, $72-\mathrm{kDa}$ type IV collagenase, neutrophil gelatinase \\
\hline MMP-3 & Simple hemopexin domain & Stromelysin-1, transin-1, proteoglycanase, procollagenase-activating protein \\
\hline MMP-7 & Minimal domain & Matrilysin, matrin, PUMP1, small uterine metalloproteinase \\
\hline MMP-8 & Simple hemopexin domain & Collagenase-1, neutrophil collagenases, PMN collagenase \\
\hline MMP-9 & Gelatin-binding & Gelatinase B, 92-kDa gelatinase, 92kDa type IV collagenase \\
\hline MMP-10 & Simple hemopexin domain & Stromelysin-2, transin-2 \\
\hline MMP-11 & Furin-activated and secreted & Stromelysin-3 \\
\hline MMP-12 & Simple hemopexin domain & Collagenase- 3 \\
\hline MMP-13 & Simple hemopexin domain & Collagenase-3 \\
\hline MMP-14 & Transmembrane & MT1-MMP, MT-MMP1 \\
\hline MMP-15 & Transmembrane & MT2-MMP, MT-MMP2 \\
\hline MMP-16 & Transmembrane & MT3-MMP, MT-MMP3 \\
\hline MMP-17 & GPI-linked & MT4-MMP, MT-MMP4 \\
\hline MMP-18 & Simple hemopexin domain & Collagenase-4 (Xenopus; no human homologue known) \\
\hline MMP-19 & Simple hemopexin domain & RASI-1, MMP-18 ¥ \\
\hline MMP-20 & Simple hemopexin domain & Enamelysin \\
\hline MMP-21 & Vitronectin-like insert & Homologue of Xenopus XMMP \\
\hline MMP-22 & Simple hemopexin domain & CMMP (chicken: no human homologue known) \\
\hline MMP-23 & Type II transmembrane & Cysteine array MMP (CA-MMP), femalysin, MIFR, MMP-21 \\
\hline MMP-24 & Transmembrane & MT5-MMP, MT-MMP5 \\
\hline MMP-25 & GPI-linked & MT6-MMP, MT-MMP6, leukolysin \\
\hline MMP-26 & Minimal domain & Endometase, matrilysin-2 \\
\hline MMP-27 & Simple hemopexin domain & \\
\hline MMP-28 & Furin-activated and secreted & Epilysin \\
\hline
\end{tabular}

*MMP-4, -5, and -6 have been abandoned. ¥ When MMP-19 was cloned it was initially called MMP-18. However, an MMP from Xenopus had alreadyreceived that designation, and therefore this MMP is now known as MMP-19. GPI, glycosylphatidylinositol; MMP, matrix metalloproteinase; MT-MMP,membrane type MMP; PMN, polymorphonuclear neutrophil; PUPM, putative metalloproteinase

MMPs are proteolytic enzymes and their basic mechanism of action regulates various cell behaviors and is involved in the tumor microenvironment, and their expression and activation is increased in various human cancers compared with normal tissue (Egeblad and Werb, 2002).

\section{The MMP family}

The MMPs are endopeptidases which can cleave several components of the ECM. The MMPs are divided into collagenases, gelatinases, stromelysins, matrilysins, membranetype (MT)-MMPs and others on the foundation of their specificity for ECM components (Nagase et al., 2006; Piperi 
and Papavassiliou, 2012; Werb, 1997). They comprise more than 21 human MMPs and numerous homologues from other species (Table 1) (Birkedal-Hansen et al., 1993; Nagase et al., 2006). The basic structure of MMPs is made up of the following homologous domains: 1) signal peptide which directs MMPs to the secretory or plasma membrane insertion pathway, 2) N-terminal pro-domain (propeptide), involved in maintaining the latency of proteins, including a highly conserved sequence RPCGVPDV, 3) catalytic domain, including the consensus HexxHxxGxxH zinc-binding motif, 4) C-terminal domain (hemopexin-like domain) which mediates interactions with substrates and confers specificity of the enzymes, 5) hinge region which links the catalytic and the hemopexin domain (Fig. 2) (Egeblad and Werb, 2002; Piperi and Papavassiliou, 2012; Sternlicht and Werb, 2001).

1) Minimal domain MMPs: MMP-7, -26

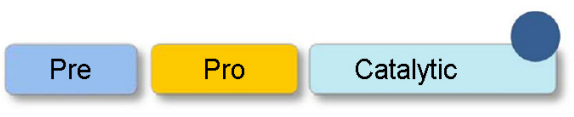

2) Simple hemopexin domain-containing MMPs: MMP-1, $-3,-8,-10,-12,-13,-18,-19,-20,-22,-27$

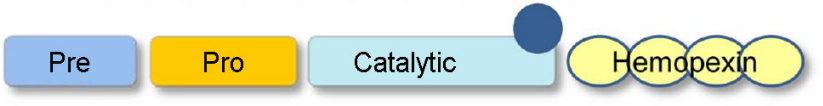

3) Gelatin-binding MMPs: MMP-2, -9

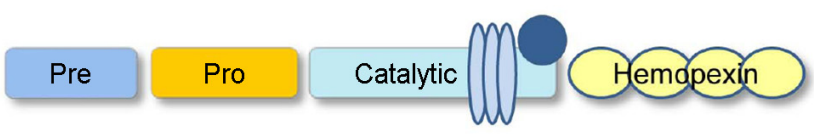

4) Furin-activated secreted MMPs: MMP-11, -28

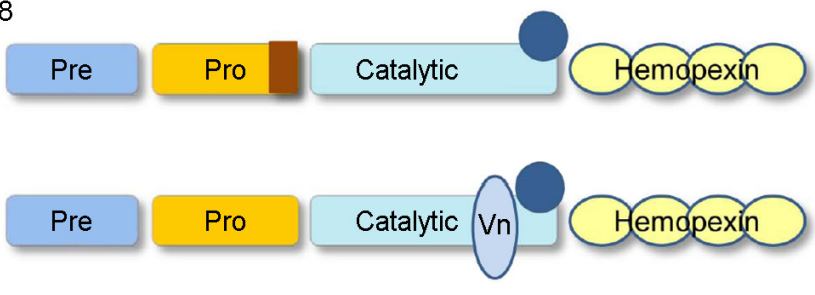

5) Vitronectin-activated domain: MMP-21

6) Transmembrane MMPs: MMP-14 (MT1-MMP), -15 (MT2-MMP), -16 (MT3-MMP), -24 (MT5-MMP)

$$
\text { Pre }
$$

Pro
Catalytic

7) GPI-linked MMPs: MMP-17 (MT4-MMP), -25 (MT6-MMP)

Pre Pro Catalytic GPI

\section{Zinc ion} Vn Vitronectin

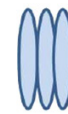

Fibronectin type II motif
Furin cleavage site

TM Transmembrane domain

Glycosylphosphatidylinositol-anchoring sequence

Fig. 2. General protein structure of the various MMPs. MMPs are multidomain enzymes that have a pro-domain, an active domain, a zinc-binding domain and a hamopexin domain. Each MMPs is categorized by domain structure. Abbreviations used: Pre = signal sequence; Pro = propeptide; Catalytic $=$ Catalytic domain; Hemopexin $=$ hemopexin-like domain; $\mathrm{TM}=$ transmembrane domain; $\mathrm{GPI}=$ glycophosphatidyl inositol-anchoring domain. 
The secreted zymogen (pro-MMPs) loses its signal peptide. Upon activation, the propeptide domain is cleaved off in several steps. The function of the propeptide is to maintain latency of the matrixins until a signal for activation is given (Birkedal-Hansen, 1995). A number of studies show that the propeptide functions by virtue of the cysteine switch mechanism, involving the coordination of Cys70-80 in the conserved sequence RPCGVPDV to the active center zinc atom (Nagase et al., 2006). But, cysteine, by itself, does not explain latency (Birkedal-Hansen et al., 1993). Cysteine residue can be blocked with iodoacetaminde and the enzyme still retains its latency (Birkedal-Hansen et al., 1993; Piperi and Papavassiliou, 2012). On the other hand, activation of the MMPs can then be achieved by moieties other than Cys to destabilize the propeptide structure or interaction with the enzyme (Piperi and Papavassiliou, 2012).

\section{Classes of MMPs inhibitors}

The major physiologic inhibitors of the MMPs are $\alpha-2$ macroglobulin and tissue inhibitors of metalloproteinases (TIMPs), proteins which specifically inhibit theses proteases and produced by many cell types (Baker et al., 2002; Brew et al., 2000). $\alpha-2$ macroglobulin inactivate susceptible proteinases by entrapment following cleavage of the bait region (Birkedal-Hansen et al., 1993; Sottrup-Jensen, 1989). The most thoroughly studied class of natural MMP inhibitors are TIMPs. The TIMP family comprises four structurally related members, TIMP-1, $-2,-3$, and -4 . The relative molecular mass range from 22 to $30 \mathrm{kDa}$. Among TIMPs families, TIMP-1 and -2 are secreted in soluble form, whereas TIMP-3 is associated with the ECM (Birkedal-Hansen, 1995; Brew et al., 2000). The TIMPs bind with high affinity in a 1:1 molar ratio to the catalytic site of active MMPs (Kahari and Saarialho-Kere, 1999). TIMPs are composed of a large N-terminal domain responsible for MMP inhibition and a smaller C-terminal domain (Brew et al., 2000). The TIMPs in general do not demonstrate specificity for any particular MMP, although TIMP-2 has been shown to show some degree of preference for MMP-2 and TIMP-1 for MMP-9 (MacDougall et al., 1999; Visse and Nagase, 2003). TIMP concentrations generally far exceed the concentration

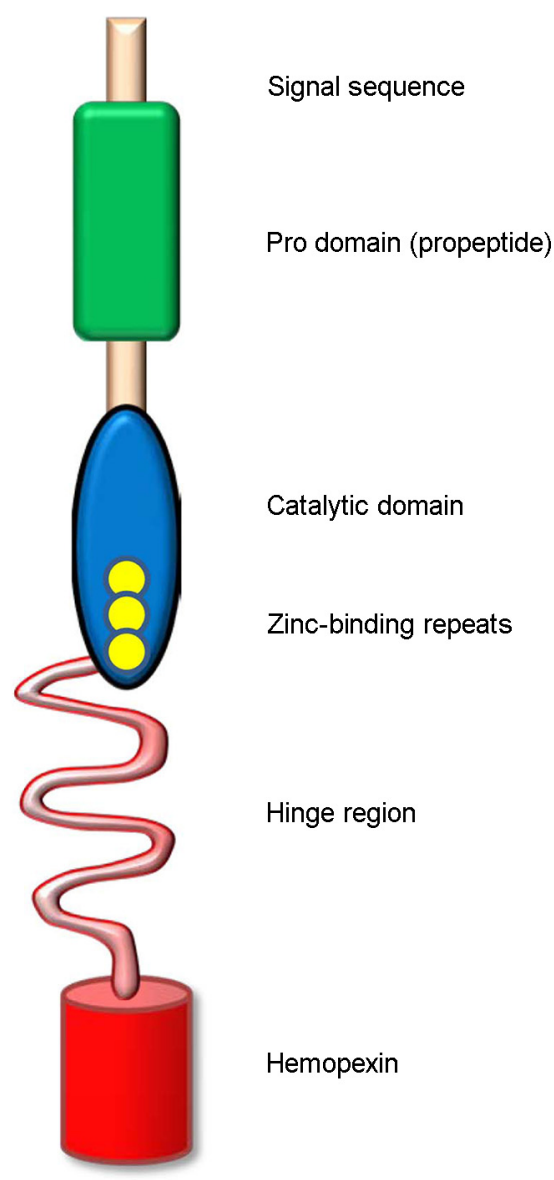

Fig. 3. Domain structure of MMP-9. The N-terminal signal sequence is followed by the propeptide domain. The catalytic site contains the essential zinc ion-binding site. A cysteine residue within the autoinhibitory propeptide domain interacts with zinc to prevent substrate binding. MMP-9 is unique from other MMPs, in that the catalytic domain contains three zinc-binding repeats (yellow circles). The zinc-binding repeats can bind to denatured collagen, enhancing its interaction with gelatin or gelatin-like substrates. The flexible proline-rich hinge region and a carboxy (C)-terminal haemopexin domain which functions in substrate recognition mediate interaction with enzyme substrates.

of MMPs in tissue and extracellular fluids, thereby limiting their proteolytic activity to focal pericellular sites (Hua and Muschel, 1996). The transcription of TIMPs is regulated by similar cytokines and growth factors that control MMP expression, that is TGF- $\beta$, TNF- $\alpha$, IL-1, and IL-6, although often in distinctive ways (Brew et al., 2000; Visse and Nagase, 2003). 


\section{MMP-9 and tumor metastasis}

Two different soluble gelatinases have been identified: gelatinase A, $72 \mathrm{kDa}$ (MMP-2), and gelatinase $\mathrm{B}, 92 \mathrm{kDa}$ (MMP-9) (Birkedal-Hansen et al., 1993). The MMP-9 gene is located in 20q11.2-q13.1 (Nagase et al., 2006). MMP-9 is a multi-domain enzyme and contains the signal peptide domain, pro-domain, catalytic domain and hemopexin domain (Fig. 3) (Egeblad and Werb, 2002; Van den Steen et al., 2002). MMP-9 is secreted as an inactive form containing the pro-domain. Pro-MMPs are activated through several different mechanisms, including proteolytic activation (MacDougall et al., 1999; Sternlicht and Werb, 2001). The catalytic domain has an active site for enzymatic activity and also contains a gelatin-binding region (Piperi and Papavassiliou, 2012). MMP-9 has many substrates including elastin, galectin3, ICAM-1 and collagens (Egeblad and Werb, 2002; Ram et al., 2006; Sehgal et al., 1998). Pro-MMP-9 can be activated by MMP-3, which cleaves pro-MMP-9 at several sites (Kupferman et al., 2000). MMP-9 influences the development of several human malignancies by, degradation of collagen IV in the basement membrane and the ECM promotes tumor progression, including invasion, metastasis, growth and angiogenesis (Morrison et al., 2001; Stamenkovic, 2000). MMP-9 is produced and secreted by many types of host stromal cells, inflammatory cells and malignant cells (Coussens and Werb, 1996; Farina and Mackay, 2014). In ovarian cancer cell lesions, it was observed that macrophage infiltration was decreased in MMP-9 deficient mice (Huang et al., 2002). In other experimental studies, expression of MMP-9 is increased in melanoma cells or fibrosarcoma cells, which resulted in markedly enhanced metastasis and invasive activity (Bernhard et al., 1995; Kupferman et al., 2000). Previous studies demonstrated that blockade of MMP-9 expression can reduce metastatic potential in metastasis of many cancers, including prostate cancer, melanomas and fibrosarcomas (Hua and Muschel, 1996; Sehgal et al., 1998). Both proteolytic removal and modification of MMP-9 are essential processing steps and involved in the malignant transition from primary tumor into invasive metastatic tumor (Fig. 1) (Birkedal-Hansen,
1995; Bjorklund and Koivunen, 2005). Increased plasma levels of soluble gelatinases correlated with higher occurrence of metastasis in many cancers and considered as an effective prognostic factor (Bjorklund and Koivunen, 2005). In previous studies, plasma and serum levels of MMP-9 were correlated with the development and extent of metastases in the lung and lymph nodes of rat mammary adenocarcinoma (Bjorklund and Koivunen, 2005; Nakajima et al., 1993; Nikkola et al., 2005). In murine xenograft models, constitutively expression of MMP-9 enhanced lung colonization in a lung metastasis model (Itoh et al., 1999; MacDougall et al., 1999).

\section{Concluding remarks}

The mechanical principle of cancer metastasis is highly complex and essential for a profound understanding of carcinogenesis. Due to the biological and technical difficulties such as multi-step processing of metastasis, complex cross communication between metastatic and host stromal cells, and difficulties in studying metastatic steps using in vivo model systems, molecular mechanisms underlying each metastatic step are still poorly understood (Egeblad and Werb, 2002). MMP-9 is one of the classic metastasispromoting genes implicated with metastasis of many types of human cancers (Coussens and Werb, 1996; Deryugina and Quigley, 2006; Stamenkovic, 2000). The basic action of MMP-9 has proven sufficiently sophisticated to orchestrate several functions. In various studies, it is suggested that MMP-9 is involved in such functional characteristics which allow them to promote many physiological remodeling processes, including metastasis. These could include actions that can be used to more effectively treat various cancers.

\section{Acknowledgements}

This research was supported by Basic Science Research Program through the National Research Foundation of Korea (NRF) funded by the Ministry of Education (NRF2013R1A1A2008633). 


\section{REFERENCES}

Baker AH, Edwards DR, Murphy G. Metalloproteinase inhibitors: biological actions and therapeutic opportunities. J Cell Sci. 2002. 115: 3719-3727.

Bernhard EJ, Hagner B, Wong C, Lubenski I, Muschel RJ. The effect of E1A transfection on MMP-9 expression and metastatic potential. Int J Cancer. 1995. 60: 718-724.

Birkedal-Hansen H. Proteolytic remodeling of extracellular matrix. Curr Opin Cell Biol. 1995. 7: 728-735.

Birkedal-Hansen H, Moore WG, Bodden MK, Windsor LJ, Birkedal-Hansen B, DeCarlo A, Engler JA. Matrix metalloproteinases: a review. Crit Rev Oral Biol Med. 1993. 4: 197 -250 .

Bjorklund M, Koivunen E. Gelatinase-mediated migration and invasion of cancer cells. Biochim Biophys Acta. 2005. 1755: 37-69.

Brew K, Dinakarpandian D, Nagase H. Tissue inhibitors of metalloproteinases: evolution, structure and function. Biochim Biophys Acta. 2000. 1477: 267-283.

Coussens LM, Werb Z. Matrix metalloproteinases and the development of cancer. Chem Biol. 1996. 3: 895-904.

Deryugina EI, Quigley JP. Matrix metalloproteinases and tumor metastasis. Cancer Metastasis Rev. 2006. 25: 9-34.

Egeblad M, Werb Z. New functions for the matrix metalloproteinases in cancer progression. Nat Rev Cancer. 2002. 2: 161-174.

Farina AR, Mackay AR. Gelatinase B/MMP-9 in Tumour Pathogenesis and Progression. Cancers (Basel). 2014. 6: 240-296.

Gialeli C, Theocharis AD, Karamanos NK. Roles of matrix metalloproteinases in cancer progression and their pharmacological targeting. FEBS J. 2011. 278: 16-27.

Groblewska M, Siewko M, Mroczko B, Szmitkowski M. The role of matrix metalloproteinases (MMPs) and their inhibitors (TIMPs) in the development of esophageal cancer. Folia Histochem Cytobiol. 2012. 50: 12-19.

Hanahan D, Weinberg RA. The hallmarks of cancer. Cell. 2000. 100: 57-70.

Hua J, Muschel RJ. Inhibition of matrix metalloproteinase 9 expression by a ribozyme blocks metastasis in a rat sarcoma model system. Cancer Res. 1996. 56: 5279-5284.

Huang S, Van Arsdall M, Tedjarati S, McCarty M, Wu W, Langley R, Fidler IJ. Contributions of stromal metalloproteinase-9 to angiogenesis and growth of human ovarian carcinoma in mice.
J Natl Cancer Inst. 2002. 94: 1134-1142.

Itoh T, Tanioka M, Matsuda H, Nishimoto H, Yoshioka T, Suzuki

R, Uehira M. Experimental metastasis is suppressed in MMP9-deficient mice. Clin Exp Metastasis. 1999. 17: 177-181.

Kahari VM, Saarialho-Kere U. Matrix metalloproteinases and their inhibitors in tumour growth and invasion. Ann Med. 1999. 31: 34-45.

Kim JW, Wong CW, Goldsmith JD, Song C, Fu W, Allion MB, Herlyn M, Al-Mehdi AB, Muschel RJ. Rapid apoptosis in the pulmonary vasculature distinguishes non-metastatic from metastatic melanoma cells. Cancer Lett. 2004. 213: 203-212.

Kupferman ME, Fini ME, Muller WJ, Weber R, Cheng Y, Muschel RJ. Matrix metalloproteinase 9 promoter activity is induced coincident with invasion during tumor progression. Am J Pathol. 2000. 157: 1777-1783.

Leber MF, Efferth T. Molecular principles of cancer invasion and metastasis (review). Int J Oncol. 2009. 34: 881-895.

Lynch CC, Matrisian LM. Matrix metalloproteinases in tumor-host cell communication. Differentiation. 2002. 70: 561-573.

MacDougall JR, Bani MR, Lin Y, Muschel RJ, Kerbel RS. 'Proteolytic switching': opposite patterns of regulation of gelatinase $\mathrm{B}$ and its inhibitor TIMP-1 during human melanoma progression and consequences of gelatinase $\mathrm{B}$ overexpression. Br J Cancer. 1999. 80: 504-512.

Morrison CJ, Butler GS, Bigg HF, Roberts CR, Soloway PD, Overall CM. Cellular activation of MMP-2 (gelatinase A) by MT2-MMP occurs via a TIMP-2-independent pathway. J Biol Chem. 2001. 276: 47402-47410.

Murphy G. The ADAMs: signalling scissors in the tumour microenvironment. Nat Rev Cancer. 2008. 8: 929-941.

Nagase H, Visse R, Murphy G. Structure and function of matrix metalloproteinases and TIMPs. Cardiovasc Res. 2006. 69: 562 -573 .

Nakajima M, Welch DR, Wynn DM, Tsuruo T, Nicolson GL. Serum and plasma M(r) 92,000 progelatinase levels correlate with spontaneous metastasis of rat 13762NF mammary adenocarcinoma. Cancer Res. 1993. 53: 5802-5807.

Nguyen DX, Massague J. Genetic determinants of cancer metastasis. Nat Rev Genet. 2007. 8: 341-352.

Nikkola J, Vihinen P, Vuoristo MS, Kellokumpu-Lehtinen P, Kahari VM, Pyrhonen S. High serum levels of matrix metalloproteinase-9 and matrix metalloproteinase-1 are associated with rapid progression in patients with metastatic melanoma. Clin Cancer Res. 2005. 11: 5158-5166.

Pantel K, Brakenhoff RH. Dissecting the metastatic cascade. Nat 
Rev Cancer. 2004. 4: 448-456.

Piperi C, Papavassiliou AG. Molecular mechanisms regulating matrix metalloproteinases. Curr Top Med Chem. 2012. 12: 1095-1112.

Ram M, Sherer Y, Shoenfeld Y. 2006. Matrix metalloproteinase-9 and autoimmune diseases. J Clin Immunol. 2012. 26: 299 -307 .

Roeb E, Matern S. [Matrix metalloproteinases: Promoters of tumor invasion and metastasis - A review with focus on gastrointestinal tumors]. Z Gastroenterol. 2001. 39: 807-813.

Sehgal G, Hua J, Bernhard EJ, Sehgal I, Thompson TC, Muschel RJ. Requirement for matrix metalloproteinase-9 (gelatinase B) expression in metastasis by murine prostate carcinoma. Am J Pathol. 1998. 152: 591-596.

Sottrup-Jensen L. Alpha-macroglobulins: structure, shape, and mechanism of proteinase complex formation. J Biol Chem. 1989. 264: 11539-11542.
Stamenkovic I. Matrix metalloproteinases in tumor invasion and metastasis. Semin Cancer Biol. 2000. 10: 415-433.

Sternlicht MD, Werb Z. How matrix metalloproteinases regulate cell behavior. Annu Rev Cell Dev Biol. 2001. 17: 463-516.

Van den Steen PE, Dubois B, Nelissen I, Rudd PM, Dwek RA, Opdenakker G. Biochemistry and molecular biology of gelatinase B or matrix metalloproteinase-9 (MMP-9). Crit Rev Biochem Mol Biol. 2002. 37: 375-536.

Vandooren J, Van den Steen PE, Opdenakker G. Biochemistry and molecular biology of gelatinase $\mathrm{B}$ or matrix metalloproteinase9 (MMP-9): the next decade. Crit Rev Biochem Mol Biol. 2013. 48: 222-272.

Visse R, Nagase H. Matrix metalloproteinases and tissue inhibitors of metalloproteinases: structure, function, and biochemistry. Circ Res. 2003. 92: 827-839.

Werb Z. ECM and cell surface proteolysis: regulating cellular ecology. Cell. 1997. 91: 439-442. 